\title{
Análise das técnicas de propulsão de atletas praticantes de modalidades em cadeira de rodas: um estudo de revisão
}

\author{
Propulsion techniques analysis of wheelchair \\ athletes: a review study
}

\author{
Allan James de Castro de Bussmann', Márcia Greguol²
}

BUSSMANN, A. J. C.; GREGUOL, M. Análise das técnicas de propulsão de atletas praticantes de modalidades em cadeira de rodas: um estudo de revisão. Rev. Ter. Ocup. Univ. São Paulo, v. 23, n. 2, p. 193-198, maio/ago. 2012.

\begin{abstract}
RESUMO: Estudos sobre o movimento humano são largamente realizados por pesquisadores que buscam a melhora do desempenho esportivo. No entanto, alguns indivíduos possuem movimentos que não se encaixam em padrões pré-estabelecidos. Pessoas com deficiência que se utilizam de cadeira de rodas para sua locomoção possuem especificidades quanto a técnica de locomoção, tanto cotidiana como no esporte. Esta revisão teve como objetivo apresentar o panorama da literatura acerca da análise das técnicas de propulsão de atletas em cadeira de rodas. Foi realizada uma busca nos portais científicos de artigos produzidos sobre o tema entre 2000 e 2011 . A busca resultou em 65 artigos, dos quais 9 atenderam aos critérios de inclusão. Os resultados apontam (a) escassez de estudos recentes; (b) os estudos selecionados enfocam a característica do movimento e de que forma esta característica influi sobre variáveis que vão desde alterações fisiológicas até o desempenho, sendo a maioria dos estudos focada na propulsão; (c) a modalidade mais pesquisada foi a corrida; (d) as imagens coletadas em sua maioria são em duas dimensões. O panorama desenhado sugere a necessidade de ampliar as pesquisas sobre o tema, ampliando os subsídios teóricos para que os treinadores tenham condições de aprimorar suas práticas e os atletas de otimizar seu desempenho.
\end{abstract}

DESCRITORES: Técnicas de exercício e de movimento/utilização; Esportes/educação; Cadeiras de rodas/utilização; Atletas; Pessoas com deficiência; Literatura de revisão como assunto.

* Parte do projeto de qualificação do mestrado de Allan James de Castro Bussmann. ${ }^{1}$ Mestrando do programa de pós-graduação associado UEL/UEM em Educação Física. ${ }^{2}$ Docente do Departamento de Ciência do Esporte - Universidade Estadual de Londrina. Endereço para correspondência: LAP/H.U - Av. Robert Koch, 60 - Vila Operária - Londrina, Paraná. Brasil. CEP:86038-350. E-mail: bussmann@uel.br 


\section{INTRODUÇÃO}

$\mathrm{O}$ homem sempre teve curiosidade em conhecer seus movimentos, de forma a aprimorar seu desempenho na prática das atividades físicas e esportivas. No entanto, nem todos os indivíduos apresentam movimentos que podem ser encaixados em padrões préestabelecidos. Pessoas com deficiência, especialmente as que se utilizam de cadeira de rodas para sua locomoção, exibem especificidades quanto às técnicas de movimento tanto nas atividades do dia-a-dia, como na prática de alguma modalidade esportiva (SANTOS; GUIMARÃES, 2002).

Apesar de existirem relatos acerca do esporte em cadeira de rodas desde a antiguidade, apenas há duas décadas esta população começou a atrair a atenção de pesquisadores, que buscam aprimorar métodos de treinamento e consequentemente o desempenho (GORGATTI; BOHME, 2003). Mara da Silva (2007) menciona que o desenvolvimento da habilidade de manejo da cadeira de rodas pelo atleta é um dos aspectos mais importantes das modalidades esportivas, uma vez que cada uma, com ou sem o uso adicional de implementos, apresenta especificidades que podem ser decisivas para a melhora do rendimento. Okana et al. (1999) destacam que, apesar de existir um consenso geral sobre estratégias no treinamento para melhorar desempenho de atletas em cadeira de rodas, poucos treinadores compreendem os aspectos técnicos que a propulsão pode trazer para a performance do atleta.

Muitas vezes existem falhas na condução técnica de movimentos em atletas em cadeira de rodas e o conhecimento destas imperfeições dá subsídio para os treinadores adaptarem e modificarem seu trabalho no sentido de corrigir falhas. Goosey-Tolfrey et al. (2010) apontam que a complexidade do esporte em cadeira de rodas é um desafio para os pesquisadores. $\mathrm{O}$ desempenho nestas modalidades depende basicamente de dois fatores - $\mathrm{o}$ atleta e a cadeira - pois é a interação entre esses elementos que permite a propulsão da cadeira de rodas e os movimentos esportivos das diversas modalidades.

No sentido de pesquisar o movimento de atletas em cadeira de rodas, cientistas vêm investigando diferentes fatores que podem influenciar a propulsão. Okawa et al. (1997), por exemplo, analisaram os diferentes fatores cinéticos que determinam a propulsão de corredores em cadeira de rodas em provas de longa distância, utilizando cinemetria para obtenção de imagens em $2 \mathrm{D}$, sendo avaliado o tempo e o ângulo de toque. Os dados apontam que o tempo de toque, tempo de ciclo e a velocidade angular não guardam relações com o tempo na corrida ou a velocidade da cadeira, no entanto o ângulo de toque tem correlação significativa com o tempo de corrida e a velocidade da cadeira. Os autores concluíram dessa forma que a habilidade de propulsão na cadeira de rodas para atletas de provas longas depende do ângulo de toque para melhor desempenho.

Assim, observa-se que, para que os profissionais tenham informações necessárias para condução de seu trabalho, fazem-se necessárias pesquisas sobre o tema, sobretudo no que se refere às técnicas recomendáveis de movimento para a propulsão da cadeira de rodas. Dessa forma, este estudo teve como escopo analisar a literatura científica a cerca da análise das técnicas de propulsão em cadeira de rodas por atletas com deficiência motora.

\section{PROCEDIMENTOS METODOLÓGICOS}

O trabalho foi iniciado pela busca de estudos bibliográficos que se propuseram a retratar a análise de movimento de atletas em cadeira de rodas. O intervalo temporal determinado foi de estudos realizados dentro do período de 2000 a 2011. Inicialmente realizou-se uma busca integrada no portal de periódicos da CAPES, onde se verificou em quais bases de dados encontravam-se artigos na temática pesquisada.

Em seguida, foram consultadas as bases de dados que possuíam os artigos, sendo elas: SportDiscus, Medline, Lilacs, Pubmed e SciELO. Foram utilizados os seguintes termos com base no problema investigado no estudo: deficiência física/ physical impairment (disability), deficiência motora/ motor impairment (disability), análise de movimento / motion analysis, cinemétria / kinemetry, biomecânica / biomechanics, esporte adaptado / adapted sport, cadeira de rodas / wheelchair, esporte paraolímpico/ paralympic sport.

Estes termos foram colocados na busca, isoladamente e, em seguida combinados em grupos de dois, três e quatro simultaneamente, tanto em português como em inglês, unidos por "e" / "and" ou "ou" / "or", a fim de uma busca mais amplificada. Na sequência, procurou-se selecionar os artigos que atenderam aos seguintes critérios de inclusão: (a) atletas com deficiência motora; (b) análise cinemática; (c) utilização de imagens; (d) análise da propulsão em cadeira de rodas; (e) publicação entre os anos de 2000 a 2011. Foram excluídas dessa revisão, monografias, dissertações e teses, vista a dificuldade de busca sistemática das mesmas.

Os estudos que atenderam completamente aos critérios de inclusão foram selecionados e posteriormente foram analisados os seguintes temas: (a) ano de publicação do estudo; (b) modalidade da amostra; (c) tipo de deficiência dos participantes do estudo; (d) instrumento utilizado para análise; (e) principais observações realizadas sobre as técnicas de propulsão da cadeira de rodas. O processo de busca, identificação e análise dos dados foram realizadas 
pelos autores do texto. Em caso de discordância, um terceiro elemento seria convidado a fazer a análise final, o que não foi necessário no presente estudo.

\section{RESULTADOS}

A busca inicial resultou em 65 artigos, porém destes apenas 9 se encaixaram nos critérios de inclusão do estudo. Entre os estudos que foram excluídos, 45 se propuseram a investigar indivíduos não atletas, três eram revisão de literatura, um tinha como foco a natação, um avaliava lançamento de dardo, quatro não estudavam propulsão, um verificava percepção de eficácia de movimento através de questionário e, por fim, um avaliava variáveis motoras na dança esportiva. Dos textos incluídos, existe um intervalo de tempo que na média é de 2,5 anos entre as publicações, sendo a mais recente publicada ano de 2011. Entre as modalidades pesquisadas, a corrida está presente na maioria dos estudos, principalmente pela característica da modalidade que facilita a coleta de dados.

Todos os estudos fizeram uso de filmagens para coleta de dados, no entanto Chow et al. (2000, 2001) utilizaram junto às imagens a técnica da eletromiografia. Já Costa et al. (2009) uniram às câmeras frequêncimetros e analisador portátil de lactato. A Tabela 1 demonstra as características dos estudos incluídos, com destaque às informações referentes ao ano de publicação, modalidade analisada, objetivo do estudo e o tamanho da amostra.

Tabela 1 - Características gerais dos estudos incluídos na revisão

\begin{tabular}{|c|c|c|c|c|c|}
\hline Autor & Ano & Modalidade & Objetivo & $\mathbf{N}$ & Resultados \\
\hline CHOW et al. & 2000 & corrida & $\begin{array}{l}\text { Analisar influência da resistência sobre } \\
\text { as características do movimento }\end{array}$ & 15 & $\begin{array}{l}\text { Apesar da diminuição da velocidade na condição } \\
\text { de grande resistência, os sujeitos mantiveram o } \\
\text { mesmo tempo e frequência de toque, com aumento } \\
\text { do tempo de toque e diminuição do tempo de } \\
\text { recuperação. }\end{array}$ \\
\hline CHOW et al. & 2001 & corrida & Comparar duas técnicas de corrida & 15 & $\begin{array}{l}\text { Existem diferenças significativas entre tempo de } \\
\text { toque e tempo de retomada entre as técnicas, além } \\
\text { de diferenças na posição do braço e na ativação } \\
\text { muscular. }\end{array}$ \\
\hline $\begin{array}{l}\text { GOOSEY- } \\
\text { TOLFREY et al. }\end{array}$ & 2001 & Corrida & $\begin{array}{l}\text { Transferência da força propulsiva para } \\
\text { a velocidade }\end{array}$ & 6 & $\begin{array}{l}\text { Os atletas aplicam mais força tangencial nos aros no } \\
\text { momento de maior velocidade }\end{array}$ \\
\hline $\begin{array}{l}\text { VANLANDEWIJCK } \\
\text { et al. }\end{array}$ & 2004 & Basquete & $\begin{array}{l}\text { Relação entre classificação funcional e } \\
\text { performance em quadra }\end{array}$ & 59 & $\begin{array}{l}\text { O desempenho na modalidade é dependente da } \\
\text { classificação. Jogadores de pontuação mais alta têm } \\
\text { melhor performance. }\end{array}$ \\
\hline KOONTZ et al. & 2005 & Indefinido & $\begin{array}{l}\text { Analisar a influência do piso na } \\
\text { propulsão }\end{array}$ & 11 & $\begin{array}{l}\text { Existem diferenças significantes entre as superfícies } \\
\text { para distância percorrida e quantidade de toques, } \\
\text { porém não há diferenças relevantes no pico de } \\
\text { velocidade entre as superfícies }\end{array}$ \\
\hline CHOW; CHAE & 2007 & corrida & $\begin{array}{l}\text { Comparar velocidade e as } \\
\text { características do ciclo de movimento } \\
\text { em diferentes fases da corrida de } 100 \\
\text { metros em cadeira de rodas }\end{array}$ & 9 & $\begin{array}{l}\text { Quando o atleta acelera para atingir sua velocidade } \\
\text { máxima, o tempo de toque e recuperação } \\
\text { diminuem, no entanto não existe diferença } \\
\text { significante entre a velocidade máxima e a } \\
\text { frequência de toque. }\end{array}$ \\
\hline COSTA et al. & 2009 & corrida & $\begin{array}{l}\text { Verificar a correlação entre frequência } \\
\text { de toque, propulsão, tamanho do aro e } \\
\text { suas influências na frequência cardíaca } \\
\text { e níveis de lactato }\end{array}$ & 52 & $\begin{array}{l}\text { Existe relação entre tamanho do aro e as variáveis } \\
\text { estudadas. }\end{array}$ \\
\hline SARRO et al. & 2010 & rugby & $\begin{array}{l}\text { Analisar a dinâmica do movimento de } \\
\text { jogadores de elite de rugby em cadeira } \\
\text { de rodas durante jogo de alto nível }\end{array}$ & 8 & $\begin{array}{l}\text { Os jogadores cobriram maior distancia e } \\
\text { tiveram maior velocidade média no primeiro } \\
\text { tempo, sendo as menores velocidades notadas } \\
\text { mais frequentemente em jogadores com menor } \\
\text { capacidade funcional. A distância coberta com } \\
\text { o cronometro acionado foi } 60 \% \text { maior do que a } \\
\text { percorrida com o tempo paralisado. }\end{array}$ \\
\hline $\begin{array}{l}\text { CRESPO-RUIZ } \\
\text { et al. }\end{array}$ & 2011 & Basquete & $\begin{array}{l}\text { Realizar um estudo piloto metodológico } \\
\text { para analisar a cinemática dos membros } \\
\text { superiores na propulsão em cadeira } \\
\text { de rodas, considerando a classificação } \\
\text { funcional de cada atleta }\end{array}$ & 10 & $\begin{array}{l}\text { Os parâmetros temporais como duração } \\
\text { do impulso, proporção da fase de impulso/ } \\
\text { recuperação, contato e ângulo de propulsão parece } \\
\text { reduzir com o aumento da classificação funcional. }\end{array}$ \\
\hline
\end{tabular}


O escopo central desta revisão foi verificar as informações existentes na literatura científica sobre a propulsão em cadeira de rodas por atletas com deficiência motora. Sobre os textos encontrados, pode-se destacar: (a) escassez de estudos recentes e um grande lapso temporal entre os trabalhos, o que pode ser justificado em parte pelas dificuldades em se obter uma amostra significativa e coletar dados em virtude das particularidades da população; (b) os estudos selecionados enfocam a característica do movimento e de que forma esta influi sobre variáveis que vão desde alterações fisiológicas até o desempenho; (c) a modalidade mais pesquisada foi a corrida, provavelmente por ser uma modalidade na qual o movimento não tem alteração de sentido, facilitando a coleta de dados e a transferência dos resultados para o cotidiano de trabalho; (d) as imagens coletadas em sua maioria são em duas dimensões, permitindo análise dentro do plano cartesiano simples, além de exigir menos material e baratear o custo da pesquisa.

É possível entre os textos identificar alguns padrões de análises referentes ao movimento, no entanto preocupando-se com aspectos diferentes, como as características e técnicas dos mesmos. Chow et al. (2001) comparam duas técnicas de corrida, uma chamada de convencional e a outra de para-backhand. Os dados foram coletados através de imagens em 3D e eletromiografia e, de posse das informações, foram analisadas características cinemáticas do movimento e ação muscular. Os resultados apontaram que a técnica convencional foi mais compacta e com menor variação de movimento articular, ao passo que a técnica de para-backhand foi mais apropriada para atletas de endurance, que são menos explosivos, uma vez que a característica angular desta técnica permite maior transmissão de força para roda.

$\mathrm{Na}$ área da análise do movimento, Chow e Chae (2007) apresentam um estudo no qual o objetivo foi comparar a velocidade e as características do ciclo de movimento em diferentes fases da corrida de 100 metros em cadeira de rodas. Os dados foram coletados com o auxílio de duas câmeras, uma posicionada nos primeiros 50 metros e a outra nos 50 metros finais. Velocidade média, duração e frequência de ciclo de movimento, tempo de contato e tempo de recuperação no aro e as características da velocidade e aceleração foram as variáveis analisadas. Os dados indicaram que os corredores preferem manter o mesmo ritmo de movimento durante toda a prova, no entanto a velocidade máxima que o atleta consegue desenvolver é importante fator no desempenho.

Ainda sobre o tema, Sarro et al. (2010) analisaram a dinâmica de movimento de jogadores de rugby, com o auxilio de duas câmeras. Os autores concluíram que os atletas tinham maior movimentação no primeiro tempo do jogo, bem como também a velocidade média mais elevada neste período do jogo. Os dados apontaram também relação entre a capacidade funcional e a velocidade média do atleta, já que as menores velocidades foram anotadas pelos jogadores com menor pontuação.

Entre os fatores que compõe o movimento, os aspectos cinéticos são de alta relevância. Goosey-Tolfrey et al. (2004) fizeram análise cinética de corredores em cadeira de rodas durante duas velocidades diferentes e cerificaram que o aumento da força propulsiva foi proporcional ao aumento da velocidade para acomodar o trabalho extra requerido. Os dados foram coletados utilizando medidores de força e uma câmera.

Em outra pesquisa na mesma linha, Chow et al. (2000) apresentam dados sobre o efeito do aumento da carga nas características do movimento, fazendo uso de imagens em 3D e eletromiografia para coleta de dados. Os autores concluíram que o aumento da carga não alterou o ciclo de movimento nem a atividade muscular, apresentando alterações apenas quando a inclinação vertical aumentava. Nesta situação houve ainda alteração no nível de atividade muscular e também no ciclo de movimento de forma proporcional ao aumento da carga. Porém, a maior parte da atividade elétrica do músculo não se alterou em todo o experimento, indicando que as alterações são mais fisiológicas que biomecânicas.

Ainda tratando de força, Koontz et al. (2005) realizaram estudo com foco na análise cinética da propulsão manual em diferentes pisos selecionados previamente, tanto em ambientes fechados bem como em abertos. Foram analisadas as seguintes variáveis: pico de força, torque na roda, força mecânica e força máxima resultante. Os resultados apontaram diferença de desempenho entre os diferentes pisos, indicando que o piso é um fator importante no momento de se avaliar habilidade de propulsão na cadeira de rodas. De fato, a maioria dos estudos sobre movimento em cadeira de rodas não indica o tipo de piso no qual a análise é feita, fator que pode influir diretamente nos resultados.

Existem pesquisas que fazem relações que extrapolam as questões biomecânicas, como é o caso de Costa et al. (2009). Em seu estudo, é determinada a relação entre frequência de movimento, tempo de contato com o aro e a velocidade da cadeira, com o uso de diferentes tamanhos de aros de propulsão e o efeito dos diferentes aros na frequência cardíaca e concentração de lactato no atleta em competições de velocidade. Os autores apontam, com base nos resultados, que a metodologia empregada no 
estudo pode ajudar o atleta a encontrar o tamanho ideal de aro, o que pode otimizar os resultados.

A importância de pesquisas com resultados consistentes e relevantes são fundamentais para extrapolação dos achados em situações práticas. Neste sentido, Vanlandewijck et al. (2004) apresentaram uma relação entre a classificação funcional e o desempenho de atletas de basquete feminino em cadeira de rodas de elite. Para tal, os jogos mundiais da modalidade foram filmados a fim de se verificar desempenho e os resultados indicaram que a performance das atletas classificados com pontuação alta foi melhor que a daqueles de pontuação mais baixa na maioria das variáveis do jogo (deslocamento em quadra, condução de bola, passe, finalização), demonstrando que o desempenho das atletas foi dependente da habilidade funcional.

Ruiz et al. (2011) também fizeram estudos no campo da comparação de capacidade funcional e da classificação de atletas de basquete. Em sua pesquisa os autores realizaram um estudo piloto, no qual buscavam analisar a cinemática do movimento do membro superior durante a propulsão na cadeira, utilizando uma câmera e uma esteira. A esteira, a cadeira e os cintos foram adequados à pontuação do atleta. Os resultados corroboraram os apresentados por Vanlandewijck et al. (2004), uma vez que os atletas com pontuação mais alta exibiram parâmetros temporais como duração do impulso, proporção da fase de impulso e recuperação, contato e ângulo de propulsão menores.

No trabalho de Van der Wode et al. (2001), os autores traçaram um panorama sobre a pesquisa acerca da propulsão em cadeira de rodas, apontando que inicialmente eram realizadas apenas pesquisas com foco na engenharia e na fisiologia. Recentemente o interesse em biomecânica surgiu, no entanto as pesquisas têm limitações metodológicas, como amostras heterogêneas e pequenas, além de problemas com a tecnologia. Os autores citam a necessidade de estimular pesquisas com o objetivo de aprimorar o conhecimento sobre o assunto. Ainda Vanlandewijck et al. (2001) apresentaram o estado da arte sobre a biomecânica da propulsão em cadeira de rodas, com atenção especial para o esporte. Os autores apontam diversas faces da pesquisa, desde a prevenção de lesões em decorrência da prática esportiva, até o aprimoramento dos equipamentos, porém é ressaltada a importância de estudos que apresentem resultados que possam ser transferidos para o cotidiano do esporte.

A presente revisão demonstra que existem poucas pesquisas sobre o tema e com enfoques variados, no entanto a união entre os achados pelos diversos pesquisadores fornece subsídios para que os problemas metodológicos, que são apontados como uma das limitações dos estudos, possam começar a ser sanados. O estudo também aponta que os autores precisam lidar com problemas no que se refere ao tamanho da mostra. A estratégia utilizada por alguns foi realizar suas coletas em competições, no entanto as pesquisas que necessitam de estrutura laboratorial no momento da coleta não permitem se valer desta dinâmica. Os resultados da revisão sugerem a necessidade de ampliar as pesquisas sobre a análise de movimento de atletas em cadeira de rodas, auxiliando o desenvolvimento das modalidades e ampliando os subsídios teóricos para que os treinadores tenham maiores condições de otimizar suas práticas e os atletas de aprimorar o desempenho desportivo.

BUSSMANN, A. J. C.; GREGUOL, M. Propulsion techniques analysis of wheelchair athletes: a review study. Rev. Ter. Ocup. Univ. São Paulo, v. 23, n. 2, p. 193-198, maio/ago. 2012.

\begin{abstract}
Studies about the human movement are largely carried out by researchers that seek improvements of sports performance. However, some individuals have movements that don't fit into pre-established standards. This review aims to present an overview of the literature about analysis of propulsion techniques for wheelchair athletes. A search was realized in databases for articles produced about the subject between 2000 and 2011, resulting in 65 articles, 9 of which met the inclusion criteria. The results indicate (a) lack of recent studies, (b) the selected studies focus on the characteristic of motion and how this characteristic influences on variables ranging from physiological changes to the performance, (c) the most widely researched modality was the race (d) the images are mostly collected in two dimensions. We suggest further research on the subject, extending the theoretical support for coaches to be able to improve their practices and athletes to optimize their performance.
\end{abstract}

KEY WORDS: Exercise movement techniques/utilization; Sports/education; Wheelchairs/ utilization; Athletes; Disabled persons; Review literature as topic. 


\section{REFERÊNCIAS}

CHOW, J. W; et al. Biomechanical comparison of two racing wheelchair propulsion techniques. Med. Sci. Sports Exerc., v. 33, n. 3, p. 476-448, 2001.

CHOW, J. W.; et al. Effect of resistance load on biomechanical characteristics of racing wheelchair propulsion over a roller system. J. Biomech., v. 33, n. 5, p. 601-608, 2000.

CHOW, J. W.; CHAE, W. S. Kinematic analysis of the 100-m wheelchair race. J. Biomech., v. 40, n. 11, p. 2564-2568, 2007.

COSTA, G. B.; RUBIO, M. P.; BELLOCH, S. L.; SORIANO, P. P. case study: effect of handrim diameter on performance in a paralympic wheelchair athlete. Adapt. Phys. Activ. Q., n. 26, p. 352-363, 2009.

CRESPO-RUIZ, B. M.; DEL AMA-ESPINOSA, A. J.; GILAGUDO, A. M. Relaiton between kinematic analysis of wheelchair propulsion and wheelchair functional basketeball classification. Adapt. Phys. Activ. Q., n. 28, p.157-172, 2011.

DIGIOVINE, C. P.; et al. Frequency analisys of racing wheelchair propulsion. IEEE Trans. Rehabil Engin., v. 8, n. 3, p. 385-393, 2000.

FLIESS-DOUER, O.; VAN DER WOUDE, L. H.; VANLANDEWIJCK, Y. C. Development of a new scale for perceived self-efficacy in manual wheeled mobility: a pilot study. J. Rehabil. Med., v. 43, n. 7, p. 602-608, 2011.

GOOSEY-TOLFREY, V. L.; FOWLER, N. E.; CAMPBELL, I. G.; IWNICKI, S. D. A kinetic analysis of trained wheelchair racers during two speeds of propulsion. Med. Engin. Phy., v. 23, n. 4, p. 259-266, 2001.

GORGATTI, M. G.; BÖHEME, M. T. S. Autenticidade científica de um teste de agilidade para indivíduos em cadeira de rodas. Rev. Paul. Educ. Física, v. 17, n. 1, p. 41-50, 2003.

KOONTZ, A. M.; et al. A kinetic analysis of manual wheelchair propulsion during start-up on select indoor and outedoor surfaces. J. Rehabil. Res. Dev., v. 42, n. 4, p. 447-458, 2005.

MARADA SILVA, E. Manejo de cadeira de rodas. Uma ferramenta importante no dia-a-dia do basquete em cadeira de rodas. Rev. Adpata, v. 2, n. 1, p. 21-27, 2007.

MEDOLA, F. O.; CASTELlO, G. L. M.; FREITAS, L. N. F.; BUSTO, R. M. Avaliação do alcance funcional de indivíduos com lesão medular espinhal usuários de cadeira de rodas, v. 2, n. 1, p. 12-16, 2009. Disponível em: <http://www.nee.ueg.br/seer/index. php/movimenta/article/view/212/186>. Citado em 20 out. 2011.

OKAWA, H.; et al. Kinetic factors determining wheelchair propulsion in marathon racers with paraplegia. Spinal Cord, $\mathrm{n}$. 37, p. 542-547, 1999.

SANTOS, S. S.; GUIMARÃES, F. J. S. P. Avaliação biomecânica de atletas paraolimpicos brasileiros. Rev. Bras. Med. Esporte, v. 8, n. 3, p. 92-98, 2002.

SARRO, K. J.; et al. Trackin of wheelchair rugby players in the 2008 Demolition Derby final. J. Sports Sci., v. 28, n. 2, p. 193$200,2010$.

VAN DER WOUDE, L. H. V.; et al. Biomechanics and physiology in active manuel wheelchair propulsion. Med. Engin. Phys., n. 23, p.713-733, 2001.

VAN DER WOUDE, L. H. V.; et al. Biomechanics and physiology in active manual wheelchair propulsion. Med. Engin. Phys., v. 23, n. 10, p.713-733, 2001.

VANLANDEWIJCK, Y. C.; et al. The relationship between functional potential and field performance in elite female wheelchair basketball players. J. Sports Sci., v. 22, n. 7, p. 668675, 2004.

VANLANDEWIJCK, Y.; THEISEN, D.; DALY, D. Wheelchair propulsion biomechanics - implications for wheelchair sports. Sports Med., v. 31, n.5, p.339-367, 2001.

Recebido para publicação: 11/05/2012

Aceito para publicação: 28/08/2012 